\title{
Simultaneous shape measurement and layers detection by means of low coherence interferometry and optical coherence tomography
}

\author{
Sławomir Tomczewski ${ }^{*}$, Anna Pakuła and Leszek Sałbut \\ Institute of Micromechanics and Photonics, Warsaw University of Technology, 8 Sw. A. Boboli St., 02-525 \\ Warsaw, Poland
}

Received May 14, 2012; accepted June 03, 2012; published June 30, 2012

\begin{abstract}
In this paper the authors show the application of low coherence interferometry and optical coherence tomography with a nonGaussian light source, which are applicable in simultaneous measurement of micromembrane and detection of layers deposited on a silicon wafer. Exemplary measurement results obtained with laboratory setup are presented.
\end{abstract}

Low coherence interferometry (LCI) is a full-field measurement technique that allows shape determination in objects with height discontinuities. The basic principle of LCI is the localization of plane which indicates the maximum intensity of fringe distribution or maximum of coherence envelope during vertical scanning of the sample.

Optical coherence tomography (OCT) is a measurement technique that can be treated as an extension of LCI. OCT allows internal structural imaging of an investigated sample with micrometer scale resolution. OCT was first demonstrated by Huang et al. in 1991 [1] as a tool for indepth measurements of human retina. Those measurements were made with the $15 \mu \mathrm{m}$ axial resolution, at a wavelength of $830 \mathrm{~nm}$. Since then, there has been rapid progress in OCT techniques and growth of its application areas. The work principle of OCT is the detection of light backscattered from the internal structure of an investigated sample. This detection is done by means of low coherence interferometry. The full-field OCT measurements were introduced by Dubois et al. in 2002 [2].

Implementation of low coherent light sources in LCI measurements, originally white light Lorentzian or Gaussian sources as light bulbs and halogen lamps [3], results in obtaining interference for very short Optical Path Differences (OPD). It also enables overcoming the greatest problem of laser interferometry, while measuring shape which involves high steps - fringe order ambiguity. Generally, fringe distribution for low coherence light is characterized by maximum intensity fringe for $\mathrm{OPD}=0$, although its slight shift may occur [4], and contrast decreases with raising OPD. For measurements performed

\footnotetext{
*E-mail: s.tomczewski@mchtr.pw.edu.pl
}

with most light sources the maximum intensity fringe is determinable, to distinguish it from the adjacent fringes but the difference in amplitude is low. Therefore, wrong analysis of measurement data may occur as a result of detector noise.

To eliminate errors derived from finding the maximum intensity fringe in the interferogram with a low signal to noise ratio, complicated numerical algorithms were implemented. Those algorithms are based on finding the coherence envelope maximum [5-6]. For such algorithms it is crucial to have the Gaussian light spectrum [6].

Applying sophisticated numerical algorithms to LCI measurements has its drawback — with full field measurement, long distance of scanning and implementing many pixel detector matrices - numerical computations become extremely time and resource consuming.

It is known that by implementing two specially matched Gaussian light sources the signal to noise ratio in the interferogram is improved [7]. By extending this idea to more sources the minimal contrast difference between the intensity of zero order fringe and others may reach $71 \%$ [8].

Applying more than one Gaussian light source or technologically combined sources as white light LEDs results in obtaining non-Gaussian coherence and hence, possibly improving the performance of zero order fringe detection algorithms. In our research we applied a centroid algorithm [9] to registered data.

The possibility of combining light sources or shaping spectral characteristics is also important in measurements performed with optical coherence tomography as the axial resolution of an OCT system is determined by spectral characteristic of a light source. If the source has the Gaussian spectral characteristic then the axial resolution can be calculated as:

$$
\Delta z=\frac{2 \ln (2)}{\pi} \frac{\lambda_{0}^{2}}{\Delta \lambda},
$$

where $\lambda_{0}$ is the central wavelength and $\Delta \lambda$ is the full width at half maximum of the light source spectral characteristic. 
In our system a white light LED was used as a light source, therefore the calculation of axial resolution with the presented formula (Eq. 1) is not possible. The axial resolution was calculated numerically on the basis of data obtained with the spectrometric measurement presented in Fig. 1. The value of axial resolution is $1.7 \mu \mathrm{m}$ in air. The minimal contrast difference, for the applied light source, between the zero order fringe and the adjacent ones is $24 \%$. For the Gaussian light source, with the spectral characteristic shown in Fig. 1 (see Fig. 5 for envelope) it would be $14 \%$ with an axial resolution of $2.45 \mu \mathrm{m}$.

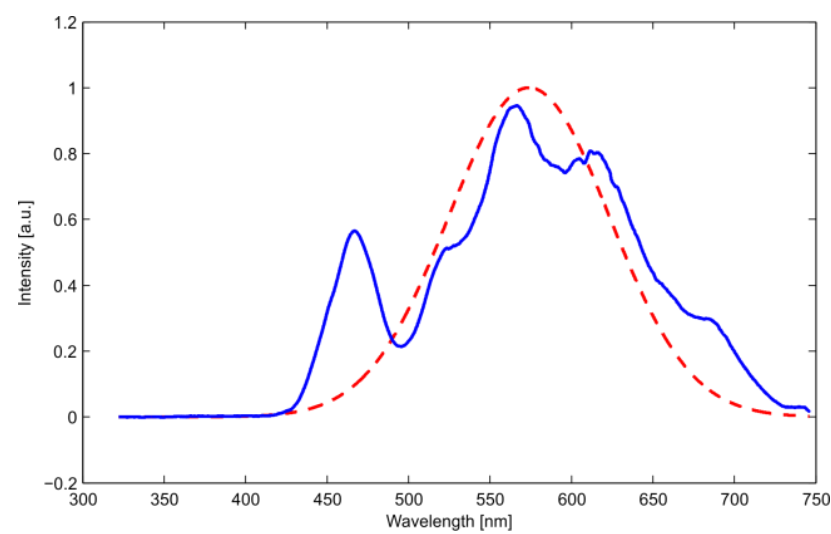

Fig. 1. Light source spectral characteristics: applied source (blue line), theoretical Gaussian (red dashed line).

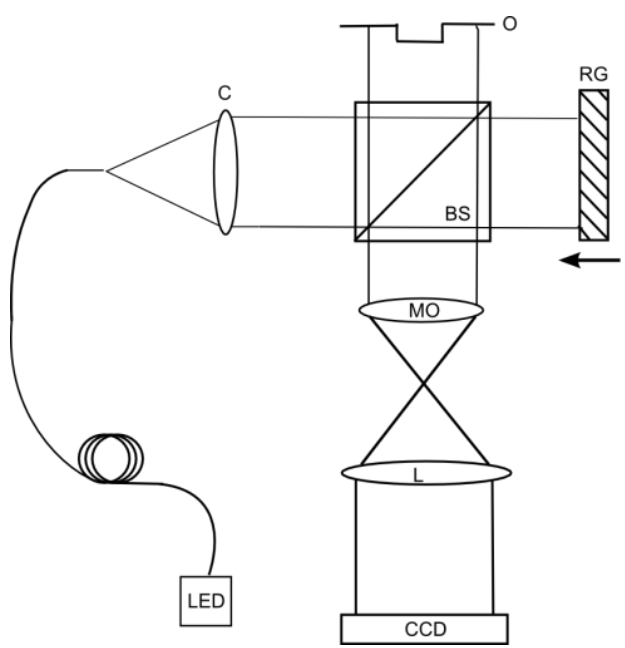

Fig. 2. Scheme of T-G interferometric setup

In our studies, full-field low coherence interferometry combined with a full-field optical coherence tomography was used to measure the membrane on a silicon wafer and visualize transparent technological layers. A detailed description of the measured sample can be found in literature [10]. The used setup is based on the TwymanGreen interferometer and its scheme is presented in Fig. 2. Low coherent light emitted from the pigtailed LED and collimated by a collimator (C) illuminates a beam splitter
(BS). The beam splitter divides the light beam into two arms: reference and sample. The light beam in the reference arm reflects from the reference glass surface (RG) placed on the piezoelectric stage and propagates back to the beam splitter. The light beam in the sample arm is reflected from the measured object $(\mathrm{O})$ and also propagates back to the beam splitter. The light beams are then combined and interfere. The intensity distribution is registered by a CCD matrix which is conjugated with the object under test by an imaging system $(\mathrm{MO}+\mathrm{L})$.

The analysis of LCI data was performed by two methods. The first method was simply finding the maximum intensity in each pixel of the matrix detector. The images were acquired with a $10 \mathrm{~nm}$ step of the piezoelectric transducer and the results from this measurement are presented in Fig. 3. The obtained height of an upper electrode over wafer surface equals $1.27 \mu \mathrm{m}$. The second method of data analysis was a centroid method and the measured electrode height was $1.25 \mu \mathrm{m}$, measurement results are presented in Fig. 4.

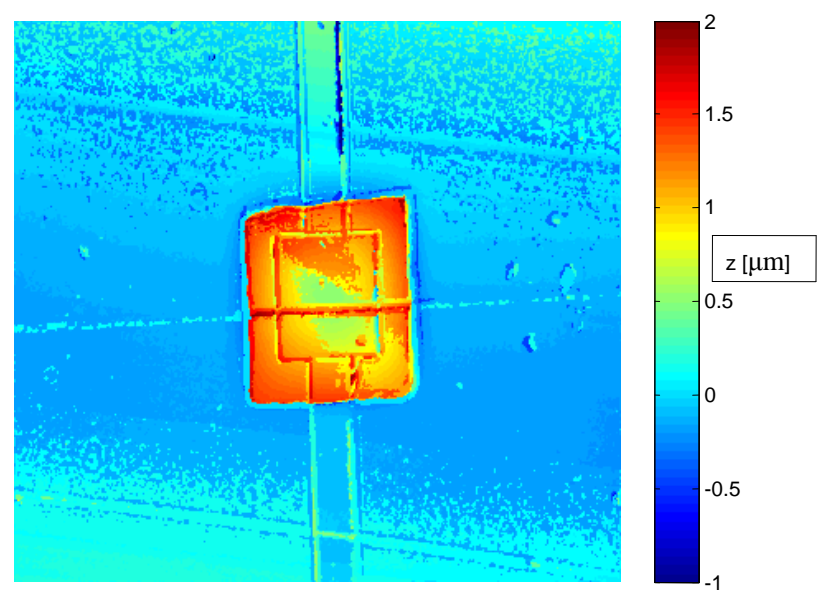

Fig. 3. Results of shape calculation by finding maximum intensity

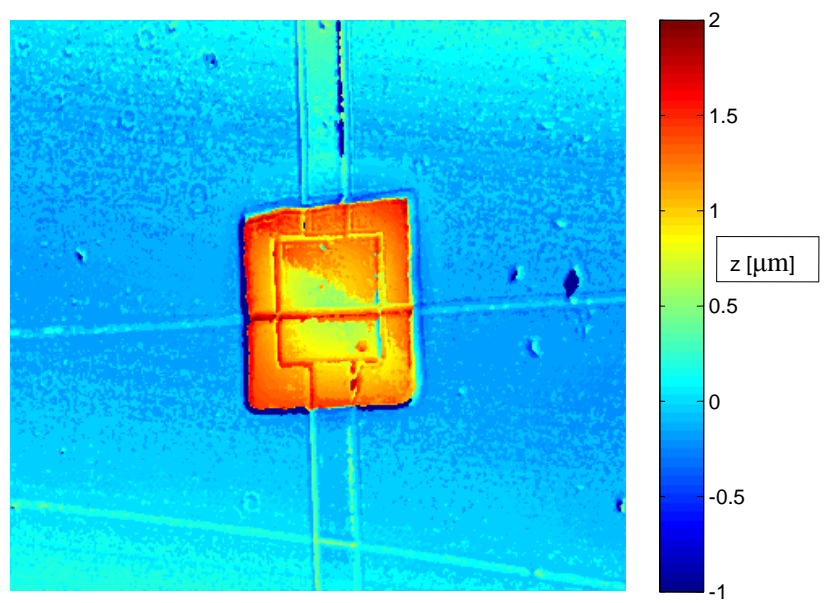

Fig. 4. Results of shape calculation with Centroid Algorithm 
Next OCT images were calculated from the acquired data. For better visualization of presented results, numerical simulation of changes in intensity and envelope of these changes calculated with the use of Hilbert transform are shown in Fig. 5. The side lobe of envelope is clearly visible in presented calculations; therefore it had to be included in interpretation of OCT measurement results.

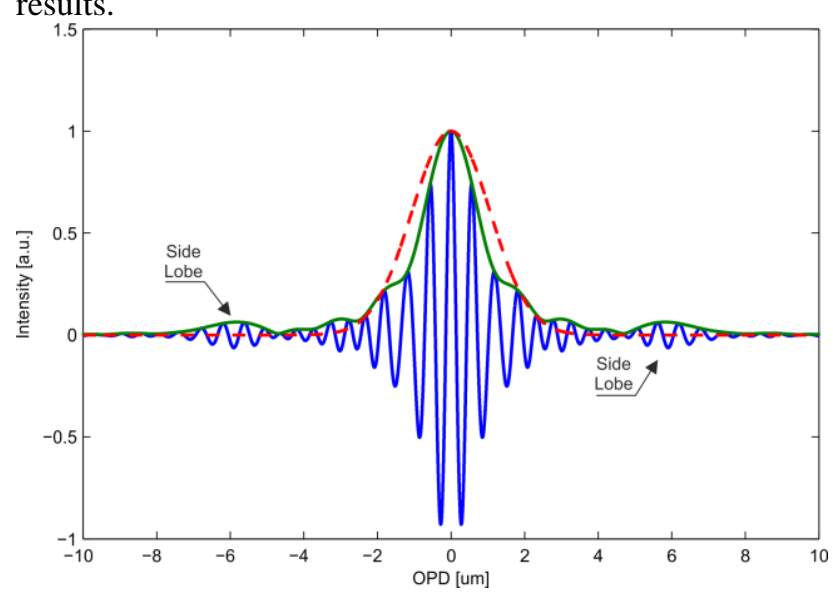

Fig. 5. Numerically calculated changes in intensity (blue line); envelope estimation (green line), theoretical envelope for Gaussian light source (red dashed line).

The results from the OCT measurements are presented in Fig. 6, as cross sections through lines AX (b) and AY (c).
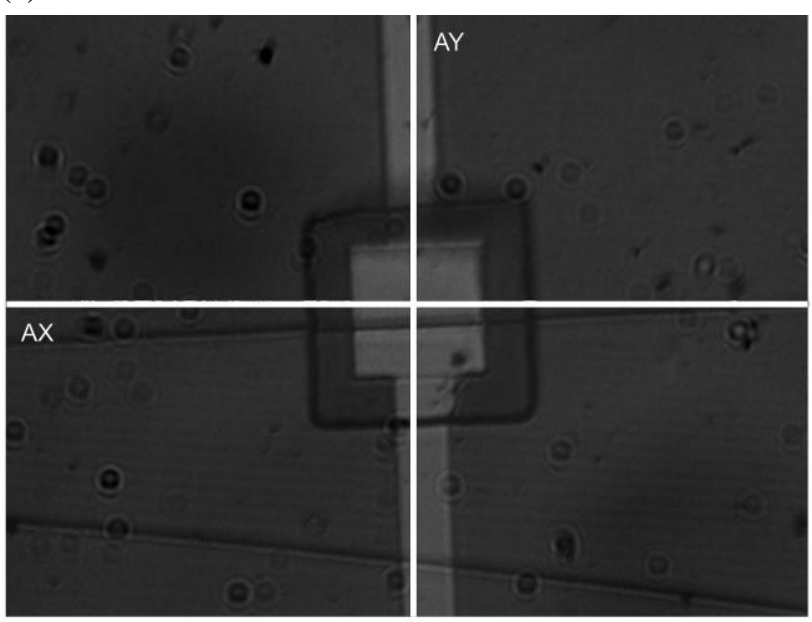

(a)

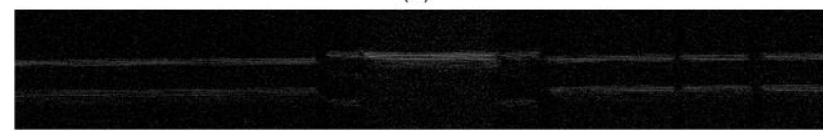

(b)

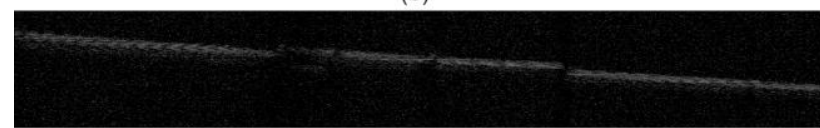

(c)

Fig. 6. OCT visualization of micromembrane on a silicon wafer, a) Front image of the measured sample with a marked position of cross sectional images; b) cross section through the AX line; c) cross section through the AY line.

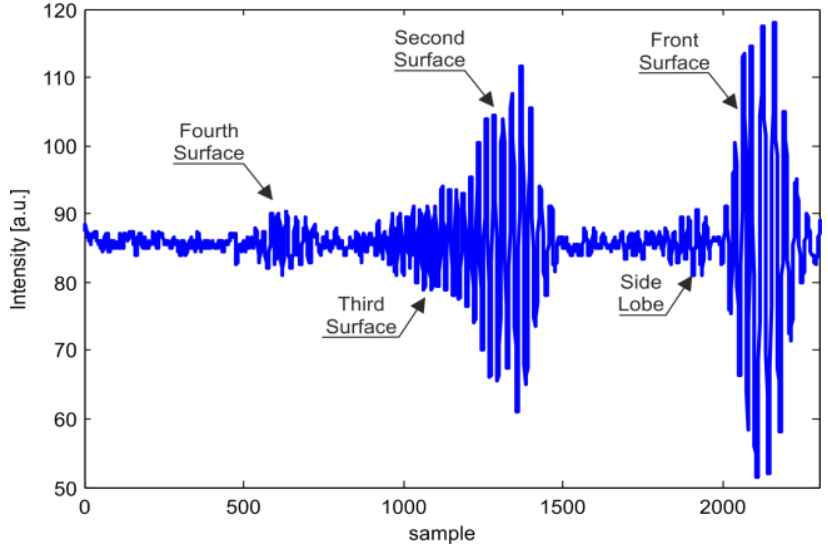

Fig. 7. Generated A-scan sample.

The cross section $\mathrm{AX}$ was done through the membrane and the silicon wafer with deposited layers. Only reflections from the first two surfaces are shown on the cross sectional image as signals from the following surfaces were considerably lower. Therefore an A-scan plot generated from acquired en-face images is presented in Fig. 7. The reflection from the first surface is clearly visible, next there is a side lobe of changes in intensity (see Fig. 5). Next, there is a signal from the second and third surface, but the small distance between those surfaces results in the overlapping of coherence envelopes. At last, the fourth surface is visible.

The cross section AY was done through the membrane and end metallic electrodes, therefore only the front surface is visible.

In this paper we presented the application of low coherence techniques (LCI and OCT) to simultaneously measure the front surface of a silicon membrane and the visualize technological layers on silicon wafer surface.

The authors acknowledge the financial support from the Faculty of Mechatronics Dean's grant nr 504 M 1143 3020000 and from the Polish Ministry of Science and Higher Education research project N N202 354135

\section{References}

[1] D. Huang et al., Science 254, 1178 (1991).

[2] A. Dubois, L. Vabre, A.C. Boccara, E. Beaurepaire, Appl. Opt. 41, 805 (2002).

[3] J.W. Goodman, Statistical Optics (John Wiley \& Sons 1985).

[4] A. Harasaki, J. Schmit, J.C. Wyant, Appl. Opt. 40, 2101 (2001).

[5] G. Kieran, J. Larkin, J. Opt. Soc. Am. A 41, 4876 (2002).

[6] A. Hirabayashi, H. Ogawa, K. Kitagawa, Appl. Opt. 41, 4876 (2002).

[7] J. Plucinski et al., Bulletin of the Polish Academy of Sciences 56, 155 (2008).

[8] A. Pakula, L. Salbut, Fringe 2009 (Springer 2009).

[9] S. Chen, A.W. Palmer, Electr. Lett. 28, 553 (1992).

[10] A. Sabac, C. Gorecki, M. Jozwki, Proc SPIE 5458, 141 (2004). 